\title{
Theta-Band Functional Connectivity and Single-Trial Cognitive Control in Sports-Related Concussion: Demonstration of Proof-of- Concept for a Potential Biomarker of Concussion
}

\author{
Ezra E. Smith AND John J.B. Allen \\ University of Arizona, Department of Psychology, Tucson, Arizona \\ (Received May 1, 2018; Final Revision November 5, 2018; Accepted November 7, 2018; First Published OnLine January 25, 2019)
}

\begin{abstract}
Objectives: This report examined theta-band neurodynamics for potential biomarkers of brain health in athletes with concussion. Methods: Participants included college-age contact/collision athletes with $(N=24)$ and without a history of concussion $(N=16)$ in Study 1. Study $2(N=10)$ examined changes over time in contact/collision athletes. There were two primary dependent variables: (1) theta-band phase-synchronization (e.g., functional connectivity) between medial and right-lateral electrodes; and (2) the within-subject correlation between synchronization strength on error trials and posterror reaction time (i.e., operationalization of cognitive control). Results: Head injury history was inversely related with medial-lateral connectivity. Head injury was also related to declines in a neurobehavioral measure of cognitive control (i.e., the single-trial relationship between connectivity and post-error slowing). Conclusions: Results align with a theory of connectivity-mediated cognitive control. Mild injuries undetectable by behavioral measures may still be apparent on direct measures of neural functioning. This report demonstrates that connectivity and cognitive control measures may be useful for tracking recovery from concussion. Theoretically relevant neuroscientific findings in healthy adults may have applications in patient populations, especially with regard to monitoring brain health. (JINS, 2019, 25, 314-323)
\end{abstract}

Keywords: EEG, Cognitive function, Head injuries, Biomarkers, Humans, Athletes

\section{INTRODUCTION}

Sports-related concussion and mild traumatic brain injury (mTBI) are increasingly visible as underdiagnosed and undertreated public health burdens (Coronado et al., 2012; Faul, Xu, Wald, \& Coronado, 2010; Langlois, RutlandBrown, \& Wald, 2006; Rutland-Brown, Langlois, Thomas, $\& \mathrm{Xi}, 2006)$. Tests that assess mild brain injury and brain functioning directly may be sensitive to changes in brain health and may be able to improve clinical prognoses as well as patient outcomes. This report examined brain functioning subsequent to concussion using theta-band functional connectivity to measure cognitive control.

\section{Cognitive Control}

Cognitive control facilitates adaptive action selection, emotion regulation, and concentration. Cognitive control is

Correspondence and reprint requests to: Ezra E. Smith, University of Arizona, Department of Psychology, 1503 E. University Blvd., Tucson, AZ 85721. E-mail: ezrasmith@email.arizona.edu analogous to the construct of executive functioning: both constructs are putatively instantiated in frontal-lobe circuitry, are important for action selection and behavioral adaptation, and may be especially vulnerable to $\mathrm{mTBI} /$ concussion (Bigler, 2007; Eierud et al., 2014; Karr, Areshenkoff, \& GarciaBarrera, 2014). Because a major component of cognitive control is adaptive behavioral responding, tasks like the Eriksen flanker, that require participants to inhibit habitual or prepotent behaviors are often used to assess individual differences in cognitive control (especially the sub-domains of response conflict or response inhibition; Zelazo et al., 2014). Moreover, patients with damage to the frontal lobes show impaired flanker performance including diminished neural indices of cognitive control, more erroneous responses, and impaired post-error slowing (PES) (e.g., Gehring and Knight, 2000; Hogan, Vargha-Khadem, Saunders, Kirkham, \& Baldeweg, 2006; Stemmer, Segalowitz, Witzke, \& Schönle, 2004; Wessel, Klein, Ott, \& Ullsperger, 2014; Ullsperger and von Cramon, 2006; Wessel et al., 2016).

PES happens when participants make an error on the flanker test, and then adapt their behavioral strategy toward slower and more cautious responding on subsequent trials. In 
healthy participants, PES has also been linked to neural activity, such that at the single-trial level a larger neural response following an error predicts greater slowing on the subsequent trial (Cavanagh, Cohen, \& Allen, 2009; Cavanagh, Meyer, \& Hajcak, 2017; Debener et al., 2005), ostensibly because that neural response reflects an instantiation of a cognitive control circuit (Cavanagh and Shackman, 2015; Cohen, 2011a; Kerns et al., 2004). More specifically, increased activity in the medial-prefrontal cortex (mPFC), as well as connectivity between the MPFC and lateral-prefrontal cortex (IPFC) predicts slower reaction times (RTs) following errors, and may implement adaptive response inhibition and cognitive control (Cavanagh and Frank 2014; Cavanagh and Shackman, 2015, Cohen, 2011a).

\section{Error-Related Theta Dynamics}

Oscillatory theta dynamics following errors are important for cognitive control inasmuch as they predict downstream cognitive control (Cavanagh et al., 2009, 2017; Cavanagh and Shackman, 2015; Cohen and Cavanagh, 2011; Van de Vijver, Ridderinkhof, \& Cohen, 2011). Event-related potentials characterized by midfrontal theta-band dynamics (e.g., the error-related negativity [ERN] and error-positivity) predict aspects of cognitive control, including PES (Gehring, Goss, Coles, Meyer, \& Donchin, 1993; Hajcak, McDonald, \& Simons, 2003), and are lower in mTBI than non-mTBI control groups (De Beaumont, Beauchemin, Beaulieu, \& Jolicoeur, 2013; Moore et al., 2015; Pontifex, O'Connor, Broglio, \& Hillman, 2009; although see Larson, Clayson, \& Farrer, 2012 for comparison). Similarly, midfrontal theta power following errors also predicts PES and cognitive control (Cavanagh et al., 2009, 2017; Van de Vijver et al., 2011), and the ERN and midfrontal theta are presumed to have a common generator (Debener et al., 2005). It is believed that theta oscillations reverberate across the brain, facilitating functional connectivity and coordination between distal brain regions (Cavanagh and Frank, 2014, Cohen, 2011a). For example, synchronous theta-rhythms between mPFC and IPFC predicts participants' cognitive control following errors (Cavanagh et al., 2009, 2017), ostensibly by facilitating communication between mPFC conflict-detection systems and IPFC response inhibition systems (Aron, Robbins, \& Poldrack, 2014; Cohen, 2011a; Kerns et al., 2004).

Despite the importance of oscillatory theta dynamics for error processing and cognitive control, the relationship between synchronous theta activity and brain injury has not been previously investigated. Because connectivity between mPFC and IPFC is important for cognitive control, it stands to reason that disrupted connectivity may also result in disrupted cognitive control (operationalized as correlations between theta-band connectivity and PES). Thus, although the ERP literature is suggestive of mTBI-induced disruption to neural activity that implements cognitive control, specifically post-error theta-band connectivity has not yet been examined in relation to mTBI (cf. Cohen, 2011a; Wessel et al., 2016). Therefore, the present report will examine whether theta-band functional connectivity between frontal brain regions can serve as a potential indicator of concussion/ mTBI.

\section{Present Report}

In Study 1, it was hypothesized that head injury would disrupt $\mathrm{mPFC}$-lPFC theta-band connectivity (operationalized as theta-band synchrony between $\mathrm{FCz}$ and F6), and that head injury would disrupt cognitive control (operationalized as the single-trial correlation coefficient between connectivity and PES). We also expected that connectivity and cognitive control would be diminished in participants with more head injuries (i.e., a dose-response relationship). Differences in behavioral performance between injured athletes and healthy athletes (e.g., accuracy, RT, PES, and response efficiency) were not expected, consistent with previous reports that found differences in neural, but not behavioral data (De Beaumont et al., 2013). It was expected that the positive correlation previously observed in healthy participants between theta-band connectivity and post-error RTs (e.g., Cavanagh et al., 2009, 2017) would be stronger in healthy athletes than athletes with any history of concussion.

In Study 2, we aimed to extend the results of Study 1 by examining within-subjects' comparisons over the course of a season of play in contact sports. A replication of the findings from Study 1 using within-subjects' analyses would more directly link alterations in theta dynamics to frontal brain health. It was hypothesized that a season of high-contact athletic play (and presumed subconcussive injury) would be associated with a decline in connectivity strength as described above for Study 1.

\section{METHODS}

\section{Participants}

For Study 1, a total of 41 student athletes were recruited from intramural contact-collision sports at a large southwestern university. Participants' concussion history was collected from athlete self-report and from certified athletic trainers that monitor athlete health. An athletic trainer conducted a clinical interview with participants as part of their admission into an intramural sports club, and participants reported concussion history to the trainer at that time. Athletes that had concussions under the supervision of the athletic trainer were interviewed again and monitored for recovery. Experimenters also administered the Post-Concussion Questionnaire (PCQ) from the Sports Concussion Assessment Tool - 2 (SCAT-2, McCrory et al., 2009) to participants before electroencephalograph (EEG) recording, and asked participants about their entire history of head injury, as well as head injury in the past 6 months (i.e., "When was the last time you were diagnosed with a concussion?"). Participant characteristics are shown in Table 1. 
Table 1. Group characteristics

\begin{tabular}{|c|c|c|c|c|c|}
\hline & $\begin{array}{c}\text { Age } \\
M(S D)\end{array}$ & $\begin{array}{l}\text { Years played } \\
M(S D)\end{array}$ & $\begin{array}{l}\text { PCQ severity } \\
M(S D)\end{array}$ & $\begin{array}{l}\text { Lifetime concussions } \\
\qquad M(S D)\end{array}$ & $\begin{array}{c}\text { Concussion last } 6 \mathrm{mo} \\
N\end{array}$ \\
\hline \multicolumn{6}{|l|}{ Study 1} \\
\hline INJ & $21.8(1.9)$ & $7.8(6.7)$ & $3.0(5.8)$ & $2.1(1.7)$ & 4 \\
\hline \multirow[t]{2}{*}{$\mathrm{AHC}$} & $21.0(1.6)$ & $4.4(5.6)$ & $3.5(4.0)$ & - & - \\
\hline & \multicolumn{3}{|c|}{$Z($ uncorrected $p)$} & & \\
\hline INJ minus $\mathrm{AHC}$ & $1.4(.17)$ & $2.0(.04)$ & $-1.2(.21)$ & & \\
\hline $\begin{array}{l}\text { Study } 2 \\
\text { (preseason) }\end{array}$ & $21.2(1.0)$ & $4.4(2.1)$ & $.6(.9)$ & $.8(.9)$ & 1 \\
\hline
\end{tabular}

There was one group of athletic-healthy-control participants (AHC, $N=16 ; 4$ females) and another group of injured (INJ) participants. Data for one of the INJ participants were not analyzed because the subject only committed one error on the flanker task (analyzed $\mathrm{N}$ for $\mathrm{INJ}=24 ; 4$ females). All other participants had at least seven useable error trials for analysis. Prior work suggests that error-related brain activity may be reliable with as few as six trials (Cavanagh et al., 2017; Olvet and Hajcak, 2009). Participants in the INJ group had a mean of 2.1 lifetime concussions $(S D=1.7$; range, 1-7). Age and concussion symptoms (i.e., PCQ total) did not statistically differ between the AHC and INJ groups.

Participants in Study $2(N=10)$ were athletes from Study 1 that had one preseason and one postseason EEG recording. These 10 participants were concussion-free across the season.

All data were collected in accord with ethical guidelines for human subject's research and under approval of an institutional review board. All athletes were provided with both written and verbal informed consent with regard to study procedures, risks/benefits, and voluntariness of participation.

\section{Procedure and Experimental Task}

Participants first completed a short interview with a trained experimenter, then completed the PCQ. EEG sensors were applied, and participants had their resting-state recording (6 min) collected before completing a variant of the flanker task. The modified Eriksen flanker task used different letter strings for different blocks (e.g., MMNMM; FFEFF; QQOQQ; VVUVV; IITII). There were a total of 320 trials distributed evenly across 8 blocks. Participants were encouraged to respond with both speed and accuracy. The flanker task was the same as the task used in ZambranoVazquez and Allen (2014) and is described in detail there (also see Supplementary Figure 1).

\section{EEG Recording and Preprocessing}

EEG was recorded from $60 \mathrm{Ag} / \mathrm{AgCl}$ scalp electrodes and two mastoid electrodes using an ElectrodeArrays EEG cap (El Paso, Tx) and Neuroscan Synamps2 amplifiers (Charlotte, NC). EEG was recorded to an online reference electrode between $\mathrm{Cz}$ and $\mathrm{CPz}$ in $\mathrm{AC}$ mode with an online bandpass filter $(.5-100 \mathrm{~Hz})$, a sampling rate of $500 \mathrm{~Hz}$, and all impedances were below $10 \mathrm{k} \Omega$. In addition, two bipolar channels were recorded to monitor horizontal and vertical ocular movements.

EEG segments with discontinuities and paroxysmal artifacts were visually identified and removed (see Smith, Reznick, Stewart, \& Allen, 2017). Data were bandpass filtered $1-100 \mathrm{~Hz}$ and notch filtered $55-65 \mathrm{~Hz}$ using a custom zero-phase shift optimal FIR filter generated following the recommendations of Cook and Miller (1992). Channels marked as bad by human raters were removed. Responselocked epochs were created $(-2500 \mathrm{~ms}$ to $2000 \mathrm{~ms})$. The data were cleaned using ICA-based methods with the FastICA toolbox and ADJUST (Mognon, Jovicich, Bruzzone, \& Buiatti, 2011). Bad channels were then interpolated using spherical splines (EEGlab v13.4.4 function eeg_interp). The artifact-free data were transformed to the current-source density montage / surface laplacian using the laplacian_perrinX function included with Cohen (2014) and based on the spherical spline approach summarized by Perrin, Pernier, Bertrand, and Echallier (1989, 1990).

Trials where participants made two errors on the same trial were excluded. For single-trial correlations between synchrony and post-error RT, only errors with RTs $>100 \mathrm{~ms}$ and only errors followed by correct trials were examined. The median number of useable error trials was 23.5 ( $S D=24.3$; range $=7-95$ ).

\section{Time-Frequency Decomposition and Functional Connectivity}

A Morlet wavelet procedure (Cohen, 2014) was used to extract the analytic signals, which are the basis for calculating inter-site phase clustering (ISPC), a measure of functional connectivity between brain regions. A family of logarithmically-spaced Morlet wavelets from 2 to $80 \mathrm{~Hz}$ was created for extraction of analytic signals; wavelet cycles were also logarithmically spaced and ranged from 3 to 10 , with lower frequencies having fewer cycles. The "theta-band" was defined as the average over 4 to $8 \mathrm{~Hz}$. ISPC indicates synchrony between time-series, and ISPC is hypothesized to 
indicate functional connectivity between brain regions. ISPC-trials is calculated as the consistency of phase angle $(\varphi)$ differences between two electrodes ( $x$ and $y$ ) for a given time-point $(t)$ and frequency $(f)$ over trials (n): $\operatorname{ISPC}_{f}=\left|n^{-1} \sum_{t=1}^{n} e^{i\left(\varphi_{x t}-\varphi_{y t}\right)}\right|$; for example, ISPC-trials indicates synchrony between electrodes that was stable at a certain TF point across many trials.

ISPC-trials coefficients for error trials were based on consistency in phase angle differences between $\mathrm{FCz}$ and $\mathrm{F} 6$ (Cavanagh et al., 2009, 2017) averaged across trials at individual TF points. We focused analyses on the relationship between medial (FCz) and right-lateral PFC (F6) because right-lateral $\mathrm{PFC}$ has been associated with response inhibition in multiple reports (see Aron et al., 2014 for review), and because previous reports have shown that the relationship between mPFC-lPFC synchrony and PES is greatest at this site (see Figure 4 in Cavanagh et al., 2009).

By comparison, ISPC-time ( $n$ in the formula above is now time points and $t$ is trial) is the consistency of several timefrequency points for a single trial, and it indicates the synchrony between electrodes for a given time period on a single-trial. This ISPC-time metric can be used for withinsubject correlations between behavior and neural activity on single-trials. Thus, ISPC-trials was used for betweensubjects' comparisons, whereas ISPC-time was used for within-subjects / single-trial correlations. A 100-ms moving window was passed over each frequency band and correlated with post-error RT, thus creating an ISPC-time series (i.e., Figure 1).

Cognitive control was operationalized as positive Spearman correlation coefficients between ISPC-time and PES. PES was operationalized as the difference between response times for one error trial (trial $\mathrm{N}$ ) and the subsequent correct trial ([trial N + 1]; Cavanagh et al., 2009); in other words, PES is the difference of post-error RTs and error RTs for pairs of single-trials. Significant correlations between PES and connectivity can result from more/less PES, more/less post-error speeding, or both depending on whether the post-error RT minus error RT differences are all positive (slowing on every post-error trial), are all negative (speeding on every post-error trial), or some combination of positive and negative (slowing and speeding on post-error trials). RTs are meaningful in this regard and can change the interpretation of a significant single-trial connectivity-RT correlation. The post-error RT minus error RT differences were evaluated for single trials and indicated that participants were most likely to demonstrate slowed responses on individual post-error trials $(68 \%$ of trials for Study 1, and $73 \%$ of trials for Study 2), suggesting that positive single trial correlations between connectivity and PES (post-error RT minus error RT; Cavanagh et al., 2009) were indicative of more connectivity predicting more slowing, whereas negative correlations were indicative of more connectivity predicting less slowing (because most trials demonstrated PES). ISPC-trials were adjusted to Rayleigh-ISPC / Rayleigh-Z $\left(N_{\text {trials }} * I S P C^{2}\right)$ to mitigate spurious inflation of ISPC due to low trial count (Cohen, 2014, p. 249).
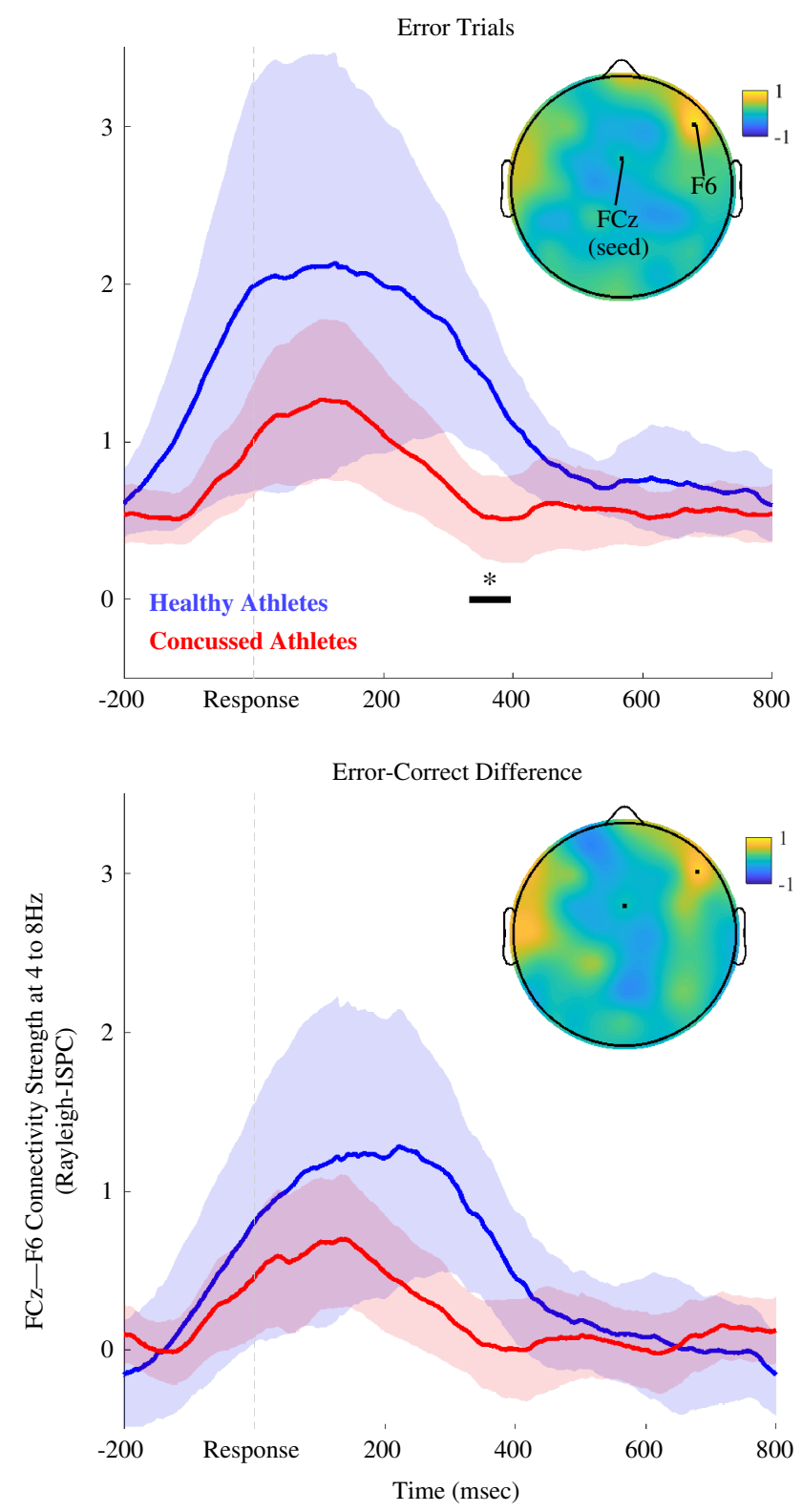

Fig. 1. Theta-band ( 4 to $8 \mathrm{~Hz}$ ) connectivity between $\mathrm{FCz}$ and F6 (Study 1). Time series of theta-band connectivity (Rayleigh ISPCtrials) between $\mathrm{FCz}$ and F6. Blue time series are data for $\mathrm{AHC}$ participants. Red time series are data for INJ participants. Shaded regions depict bootstrapped $95 \%$ confidence intervals for group means. Black bar and asterisk indicate times where connectivity significantly differed between groups (FDR-corrected $p<.05$ ). Topoplot depicts AHC-INJ difference for ISPC using a FCz seed, and the location of F6 is also noted. Top panel shows results for error trials, and bottom panel shows results for error-minus-correct difference. AHC participants were characterized by more FCz-F6 connectivity than INJ participants.

\section{Statistical Analysis}

Statistics were computed over time series $(-200 \mathrm{~ms}$ to $800 \mathrm{~ms}$ ) of theta-band synchrony (i.e., $4 \mathrm{~Hz}$ to $8 \mathrm{~Hz}$ ISPC, Figure 1) extracted from TF decomposition. The falsediscovery rate method (FDR; Benjamini and Hochberg, 1995) was used to correct for multiple comparisons over time 
series (e.g., several hundred time points). FDR-corrected $p$-values smaller than .05 were considered statistically significant. Maximum $Z$-scores from Wilcoxon rank-sum tests are reported for AHC versus INJ contrasts. Wilcoxon signedrank tests were calculated for one-sample tests (connectivityPES correlations difference from zero), and for preseason versus postseason contrasts.

Spearman rank-order correlations were calculated to measure associations between continuous variables (e.g., number of lifetime concussions, years played, and age, with FCz-F6 connectivity; see Supplementary Figure 2). A robust multiple regression analysis (fitlm function in Matlab 2017a with default robust fitting option, i.e., iteratively reweighted least squares) was used to calculate $R^{2}$. Then, significance testing for an incremental improvement in $R^{2}$ (i.e., hierarchical regression) was calculated according to the recommendations of Cohen and Cohen $\left(1983 ; F=\frac{\left(R_{Y \cdot A B}^{2}-R_{Y, A}^{2}\right) / k B}{\left(1-R_{Y, A B}^{2}\right) /\left(n-k_{A}-k_{B}-1\right)}\right)$. Data were natural log-transformed before effect size calculation (Cohen's $d$ ) to mitigate effects of outliers and skew.

\section{STUDY 1 RESULTS}

\section{Behavior}

Behavioral performance was similar across groups. RTs, accuracy, PES (post-error RT minus error RT; e.g., Cavanagh et al., 2009), and response efficiency (median response time / percentage of accurate trials, lower numbers indicate greater efficiency) were similar between AHC and INJ participants, and are shown in Table 2.

\section{Functional Connectivity}

Figure 1 shows post-error $\mathrm{FCz}-\mathrm{F} 6$ connectivity for the theta band. As expected, theta-band ISPC-trials between medial $(\mathrm{FCz})$ and lateral frontal (F6) electrodes was diminished for INJ compared to AHC on error trials $(Z=3.41$; corrected $p=.044$, uncorrected $p<.001 ; d=1.01$ at $356 \mathrm{~ms}$ ), and for the error-minus-correct difference $(Z=2.72$; corrected $p>.10$; uncorrected $p=.007 ; d=.455$ at $346 \mathrm{~ms}$ ). ISPC for error trials was not significantly related to the number of lifetime concussions or years played for INJ participants; ISPC was also unrelated to athletes' age or years played across the entire sample (see Supplementary Figure 2).

The ISPC results for the group contrast (ISPC on error trials for AHC vs. INJ groups) were similar $(Z=3.05$; corrected $p=.063$; uncorrected $p=.002 ; d=.997$ at $350 \mathrm{~ms}$ ) when examining only participants that had at least 20 error trials (AHC, $N=10$; INJ, $N=19$ ).

As an operationalization of cognitive control, the singletrial correlation coefficients between ISPC-time and posterror RT were examined. After correcting for multiple comparisons, statistics were at trend-level for single-trial correlations in $\mathrm{AHC}(Z=1.91$; corrected $p>.10$; uncorrected $p=.056 ; d=2.19$, at $582 \mathrm{~ms})$ and INJ participants $(Z=-1.66 ; \quad$ corrected $p>.10 ;$ uncorrected $p=.098$; $d=2.41$; at $306 \mathrm{~ms}$ ). There was a trend for AHC participants to demonstrate greater cognitive control than INJ participants $(Z=1.67$; corrected $p>.10$; uncorrected $p=.095 ; d=.19$ at $310 \mathrm{~ms})$.

It may be the case that INJ participants are characterized by reduced connectivity across all electrode pairs or that diminished FCz-F6 connectivity is not specific to the functioning of a hypothesized right-frontal $\mathrm{mPFC}-\mathrm{IPFC}$ circuit. Hierarchical regression and a control variable (e.g., FCz-CP4 connectivity) can test for this possibility. CP4 was selected as a control electrode in prior work (Cavanagh et al., 2009), and CP4 and F6 are equidistant with FCz. FCz-F6 peak post-error connectivity (i.e., at $356 \mathrm{~ms}$ ) remained a significant predictor of group membership after accounting for $\mathrm{FCz}-\mathrm{CP} 4$ connectivity $\left(F(2,35)=4.00 ; p=.027 ; \Delta R^{2}=.16\right)$.

A separate regression showed FCz-F6 connectivity predicted group membership after accounting for FCz-F5 connectivity $\left(F(2,35)=3.13 ; p=.056 ; \Delta R^{2}=.13\right)$, concordant with the notion that FCz-F6 phase synchrony denotes activa-

Table 2. Behavioral performance

\begin{tabular}{|c|c|c|c|c|c|}
\hline & $\begin{array}{c}\text { Total errors } \\
M(S D)\end{array}$ & $\begin{array}{c}\text { Error RT } \\
M(S D)\end{array}$ & $\begin{array}{l}\text { Accuracy } \\
M(S D)\end{array}$ & $\begin{array}{c}\text { Response efficiency } \\
M(S D)\end{array}$ & $\begin{array}{c}\text { PES } \\
M(S D)\end{array}$ \\
\hline \multicolumn{6}{|l|}{ Study 1} \\
\hline INJ & $39.1(26.5)$ & $362.3(64.3)$ & $81.3(11.1)$ & $4.9(0.7)$ & $53.9(127.3)$ \\
\hline \multirow[t]{2}{*}{$\mathrm{AHC}$} & $27.4(19.7)$ & $384.4(88.2)$ & $85.2(8.4)$ & $4.8(0.5)$ & $64.0(137.9)$ \\
\hline & \multicolumn{5}{|c|}{$Z($ uncorrected $p)$} \\
\hline INJ minus AHC & $1.57(.12)$ & $-0.55(.58)$ & $-1.04(.30)$ & $0.12(.90)$ & $-.9(.4)$ \\
\hline \multicolumn{6}{|l|}{ Study 2} \\
\hline Preseason & $43.8(25.6)$ & $349.8(64.1)$ & $79.8(10.7)$ & $4.8(.5)$ & $59.1(115.3)$ \\
\hline \multirow[t]{2}{*}{ Postseason } & $46.2(23.1)$ & $332.7(29.4)$ & $77.3(8.4)$ & $4.7(.4)$ & $48.8(95.7)$ \\
\hline & \multicolumn{5}{|c|}{$Z($ uncorrected $p)$} \\
\hline Pre minus Post & $-1.0(.33)$ & $.6(.54)$ & $1.3(.20)$ & $.7(.51)$ & $0.4(.7)$ \\
\hline
\end{tabular}


tion of a right-lateralized mPFC-IPFC circuit important for self-monitoring and response inhibition (Aron et al., 2014; Cavanagh and Shackman, 2015; Kerns et al., 2004). Supplementary Figures 3 and 4 also show that, although FCz-F6 connectivity accounted for unique variance predictive of INJ participants, there is some overlapping variance for connectivity between different pairs of electrodes.

Participants in the AHC group had fewer years played in contact-collision sports $(Z=-2.04 ; p=.04)$ than the INJ group, and years played and lifetime concussion history were correlated $(r(39)=.40 ; p=.01)$, suggesting that years played could be a potentially confounding third variable (see Table 1). Hierarchical regression was used to assess the predictive contribution of group status above and beyond number of years played. After accounting for years played, group status was still a significant predictor of peak FCz-F6 connectivity on error trials $(F(2,35)=5.88$; $\left.p=.006 ; \Delta R^{2}=.24\right)$.

\section{STUDY 2 RESULTS}

\section{Study 2: Pre- vsersus Postseason ISPC Behavior}

Accuracy, RTs, PES, and response efficiency were unchanged pre- versus postseason ( $p$ s $>.05$; see Table 2).

\section{Functional Connectivity}

After correcting for multiple comparisons, there were no significant differences in ISPC or connectivity-PES correlations for preseason versus postseason recordings. Figure 2 shows that there were trends for greater post-error ISPC at preseason compared to postseason $(Z=1.38$; corrected $p>.10$; uncorrected $p=.169 ; d=.574$ at $466 \mathrm{~ms}$ ), as well as greater error-modulated ISPC (the error-correct difference) for preseason recordings $(Z=2.09$; corrected $p>.10$; uncorrected $p=.036 ; d=.490$ at $518 \mathrm{~ms}$ ).

There were trends for connectivity to predict less PES at preseason $(Z=-1.99 ;$ corrected $p>.10 ;$ uncorrected $p=.047 ; d=3.34$ at $476 \mathrm{~ms}$ ), and for connectivity to predict greater PES at postseason $(Z=2.80$; corrected $p=.089$; uncorrected $p=.005 ; d=3.12$ at $396 \mathrm{~ms})$. Athletes tended to show a more negative correlation between connectivity and post-error RT in the preseason than postseason $(Z=-2.80$; corrected $p>.10$; uncorrected $p=.005 ; d=.158$ at $408 \mathrm{~ms}$ ), indicating that connectivity strength predicted less PES in preseason recordings, whereas connectivity predicted more PES in postseason recordings.

\section{DISCUSSION}

\section{Findings and Context}

Study 1 showed that connectivity between mPFC and IPFC was lower in athletes with any history of concussion
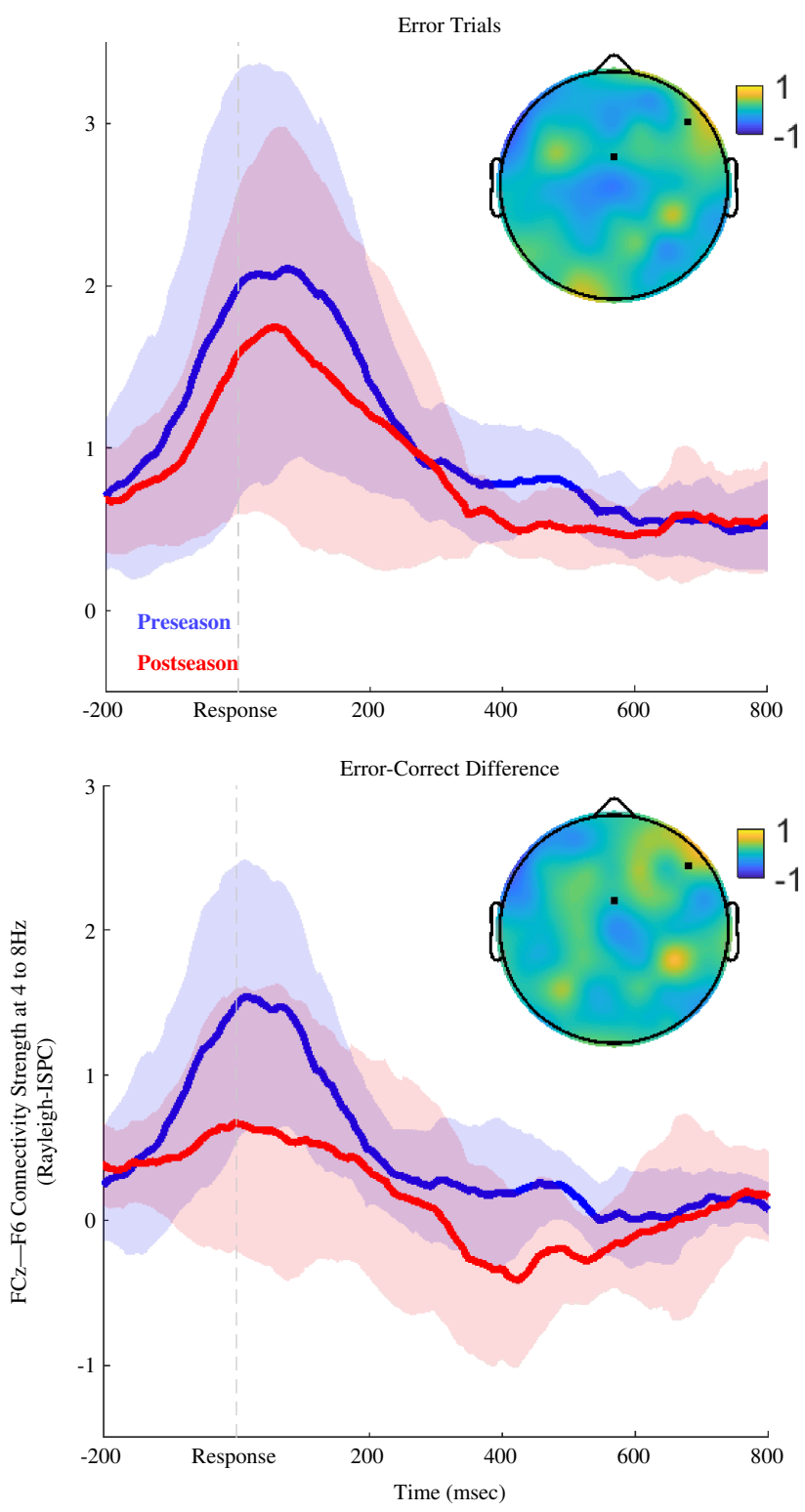

Fig. 2. Theta-band (4 to $8 \mathrm{~Hz}$ ) connectivity between $\mathrm{FCz}$ and $\mathrm{F} 6$ (Study 2). Time series of theta-band connectivity (Rayleigh ISPCtrials) between $\mathrm{FCz}$ and $\mathrm{F6}$. Blue time series are preseason data. Red time series are postseason data. Shaded regions depict bootstrapped $95 \%$ confidence intervals for condition means. After multiple comparisons corrections (FDR-method), there were no significant differences between the groups (corrected $p \mathrm{~s}>.10$ ). Topoplot depicts preseason-postseason difference for ISPC using a $\mathrm{FCz}$ seed, and the location of F6 is also noted. Top panel shows results for error trials, and bottom panel shows results for errorminus-correct difference. At preseason there was a trend for participants to show stronger FCz-F6 connectivity than at postseason.

compared to a healthy athletic cohort. There was a similar pattern of results for Study 2: athletes showed a trend-level decrease in connectivity following a season of contact/collision play even though no athletes in Study 2 were diagnosed with a concussion over the course of the season.

These studies provide initial evidence that a theorized cognitive control circuit may be useful as a measure of brain 
health. Participants with concussion/mTBI were characterized by diminished mPFC-IPFC theta-band connectivity, circuitry important for implementing cognitive control, following errors compared to athletic control participants. There were also trends for diminished modulation of brain activity following errors (i.e., error-minus-correct difference score), and altered relationships between brain activity and response inhibition (i.e., single-trial relationships between connectivity and PES). These group differences in connectivity and cognitive control emerged late in the time series $(>300 \mathrm{~ms})$ and may indicate that complex cognitive processes such as error awareness (Nieuwenhuis, Ridderinkhof, Blom, Band, \& Kok, 2001) and learning from errors (Cavanagh, Frank, Klein, \& Allen, 2010; Cohen and van Gaal, 2013) may be especially vulnerable to concussion and functioning of cortical-cortical pathways (e.g., Miller, Hayes, Lafleche, Salat, \& Verfaellie, 2017; Orr et al., 2016).

Moreover, FCz-F6 connectivity demonstrated some specificity with regard to prediction of mTBI (e.g., Supplementary Figures 3 and 4), suggestive that FCz-F6 connectivity assesses functioning of a vulnerable right-lateralized frontal lobe network that is important for behavioral inhibition (Aron et al., 2014). Yet, there was also some overlap between connectivity among electrode pairs, supportive of the notion that theta-band connectivity can facilitate simultaneous information flow between multiple brain regions (e.g., Buzsáki, 2006; Cavanagh and Shackman, 2015). Although the connectivity-PES single-trial correlation differentiated AHC and INJ groups at a trend level in Study 1, the singletrial correlations between PES and synchrony were not significantly different from zero for either group in Study 1. Van de Vijver and colleagues (2011) also reported a null singletrial connectivity-PES relationship in healthy participants, and it may be the case that the connectivity-PES relationship is characterized by a low signal-to-noise ratio and may be difficult to detect without numerous error trials.

The connectivity-PES correlation was in the opposite of expected direction for Study 2 (a more positive connectivityPES relationship in postseason recordings than preseason recordings), indicating that participants were likely to demonstrate less PES following strong mPFC-IPFC post-error connectivity. Other reports have also observed positive correlations between ISPC and post-error RTs (Cavanagh et al., 2017), and it is likely the case that participants adopt different strategies to complete the task. For example, participants demonstrating post-error speeding may be using a proactive inhibitory strategy, whereas participants demonstrating PES may be using a reactive inhibitory strategy (Buzzell et al., 2018; Cavanagh et al., 2017; Narayanan, Cavanagh, Frank, \& Laubach, 2013). Moreover, compensatory mechanisms, effort, anxiety, or depression (see Cavanagh et al., 2017; Gehring et al., 1993; Hill, Samuel, \& Foti, 2016; and Olvet, Klein, \& Hajcak, 2010 for examples of moderators of error-related brain activity) can also modulate mPFC-IPFC connectivity and may have contributed to the pattern of results in this study.

Alternatively, postseason EEG recordings would have been relatively recent to any unreported injuries (at most within 6 months) during the season, and connectivity is sometimes stronger for the first several days and weeks postinjury (Eierud et al., 2014). These potential moderators, along with a limited range of injuries, may explain why there was no apparent relationship between theta-band connectivity and lifetime number of concussions or years played (e.g., there was no apparent dose-response relationship). Overall, the results suggest that theta dynamics may be ripe for investigation especially with regard to processing efficiency in neural systems important for cognitive control and learning from mistakes.

The present results align with an emerging consensus that late-stage neural processes $(>200 \mathrm{~ms}$ post stimulus or response) important for cognitive processes like monitoring, adaptation, and error awareness (see Polich, 2007) reliably correlate with behavioral performance and concussion/mTBI. Specifically, mTBI participants frequently demonstrate evoked potentials that are delayed and weaker compared to peers (Broglio, Pontifex, O'Connor, \& Hillman, 2009; Duncan, Kosmidis, \& Mirsky, 2003; Larson, Farrer, \& Clayson, 2011). Yet only a few reports have examined group differences in response-locked functional connectivity, for example, during set-shifting (Pang, Dunkley, Doesburg, da Costa, \& Taylor, 2016), target detection (Reches et al., 2017), working memory (Bailey et al., 2017; Kumar, Rao, Chandramouli, \& Pillai, 2009), and learning tasks (Tsirka et al., 2011).

Specifically, Tsirka and colleagues (2011) reported correlations between graph theoretic metrics of functional connectivity and cognitive performance, finding that diminished clustering of theta-band connectivity in mTBI participants predicted diminished recognition memory. The results of Tsirka et al. (2011) and the present study align with a theoretical model of theta-band activity as a hub-like signal that facilitates cognitive control (e.g. Cavanagh, Zambrano-Vazquez, \& Allen, 2012; Cohen, 2011a; Cohen \& van Gaal, 2013; Wessel, 2018). Overall, the present results fit with a literature that has characterized synchronous theta dynamics as important for cognitive control and behavioral adaptation, and has demonstrated that error-related neural activity may be sensitive to neural function following mTBI.

\section{Limitations}

This study was limited in scope, and findings should be interpreted as exploratory or "proof-of-concept." Sample size and characterization, study design (i.e., cross-sectional vs longitudinal data), and behavioral testing can all be improved in future investigations. In this regard, replication and extension of the present findings are warranted before strong claims can be made regarding study hypotheses. Nonetheless, the present work is interesting and supports continued investigation.

The study was particularly limited in terms of testing participants with a mild and restricted range of injury, narrow demographics, very few psychological or medical data, and 
no paper-and-pencil neuropsychological measures. For example, factors like time since injury, severity, chronicity, medication, effort, strategy, mood, and/or premorbid conditions (e.g., ADHD, neurodevelopmental disorder, pain, depression, impulsivity, reduced processing speed), could be more likely in the INJ group, potentially confounding results and interpretation. In particular, it may be the case that preexisting factors more prevalent in the INJ group, that is, developmental disability and impulsivity, contribute to diminished connectivity/ISPC and put participants at greater risk of getting a concussion (Iverson et al., 2016). It may also be the case that less-than-ideal characterization of head injury history contributed to null dose-response correlations because of noise in self-reports, or self-reports that are confounded by impression management or over/under-reporting. Accounting for these potential moderators in a comprehensive longitudinal study that includes a detailed head injury interview and objective measures of brain structure will be important for future work.

Practice effects in Study 2 are another potential limitation: it is unclear to what extent repeated testing accounted for changes in neurobehavioral performance. Yet, there were no significant improvements in behavioral performance (i.e., practice effects) pre versus postseason, and practice effects would not account for the between-subjects' differences observed in Study 1. Moreover, practice effects for the flanker test trend towards small effect sizes over a 2-week interval ( $d=.27$ in Zelazo et al., 2014). Nonetheless, an important goal for future work will be to follow cohorts of high-risk participants over days and weeks to characterize the stability of connectivity and connectivity-PES metrics.

The present study was also limited in terms of connectivity estimation: low trial counts can inflate ISPC-based measures of connectivity. Yet, results were relatively unchanged when only examining participants with at least 20 trials, commensurate with trial counts from a previous report (i.e., Cavanagh et al., 2009). The observation that neither AHC nor INJ participants' connectivity-PES correlations differed from zero complicates any interpretation of group differences. On one hand, the flanker test is frequently used for cueing error related brain activity and is comparable with a large amount of published literature (Cavanagh et al., 2009; Olvet and Hajcak, 2009; Zelazo et al., 2014). On the other hand, future work should consider using response inhibition tasks that elicit higher error rates, and that are otherwise similar to the flanker test, as it would improve signal-to-noise ratio for connectivity estimation. Aggregate data from multiple response inhibition tests would also improve characterization and reliability of measures of synchronous brain activity in future reports (e.g., Cavanagh et al., 2012; Riesel, Weinberg, Endrass, Meyer, \& Hajcak, 2013).

\section{CONCLUSION}

Concussion was associated with weaker theta-band connectivity in a brain circuit hypothesized to be important for cognitive control and response inhibition in a limited study of college athletes. The results align with previous work showing that theta-band connectivity in an MPFC-IPFC network is activated during tasks that necessitate cognitive control. The present results are also suggestive that interarea connectivity and connectivity-PES relationships may be sensitive indicators of cognitive control inefficiency following an mTBI. It is hypothesized that these findings result from disruption in phase-based coordination of cell assemblies that depend on white matter health/integrity in the frontal lobes (Cohen, 2011a, 2011b; Fries, 2005; Sponheim et al., 2011).

These results demonstrate proof-of-concept for theoretically guided neurocognitive metrics, but are qualified by several limitations in study design and available data. Disrupted theta dynamics occurred at a later point in the time series than expected, putatively during error awareness and working memory updating; one interesting hypothesis for a future report might be that disrupted late-latency $(>300 \mathrm{~ms})$ theta dynamics in mTBI participants mediates rates of improvement in behavioral performance over trials (e.g., learning rate). Overall, the present results support the continued investigation of brain connectivity measures as potential tools for assessing brain health and cognitive functioning.

\section{ACKNOWLEDGMENTS}

Many thanks to the athletes who participated in the study; the project would not have been possible without them. Also, special thanks to the Dianne Goodridge, ATC, for her support of the project and her support of student-athletes. Finally, several research assistants were crucial to the completion of this project, especially Margaret Tobias, Jamie Velo, and Kenia Carrera. This work was funded by the National Collegiate Athletics Association (NCAA) and the Graduate and Professional Student Council of the University of Arizona. None of the authors have potential conflicts of interest to be disclosed.

\section{SUPPLEMENTARY MATERIAL}

To view supplementary material for this article, please visit https://doi.org/10.1017/S135561771800108X

\section{REFERENCES}

Aron, A.R., Robbins, T.W., \& Poldrack, R.A. (2014). Inhibition and the right inferior frontal cortex: one decade on. Trends in cognitive sciences, 18(4), 177-185.

Bailey, N.W., Rogasch, N.C., Hoy, K.E., Maller, J.J., Segrave, R.A., Sullivan, C.M., \& Fitzgerald, P.B. (2017). Increased gamma connectivity during working memory retention following traumatic brain injury. Brain Injury, 31(3), 379-389.

Benjamini, Y., \& Hochberg, Y. (1995). Controlling the false discovery rate: A practical and powerful approach to multiple testing. Journal of the Royal Statistical Society. Series B, Statistical Methodology, 57, 289-300.

Bigler, E.D. (2007). Anterior and middle cranial fossa in traumatic brain injury: Relevant neuroanatomy and neuropathology in the 
study of neuropsychological outcome. Neuropsychology, 21(5), 515.

Broglio, S.P., Moore, R.D., \& Hillman, C.H. (2011). A history of sport-related concussion on event-related brain potential correlates of cognition. International Journal of Psychophysiology, 82 (1), 16-23.

Broglio, S.P., Pontifex, M.B., O'Connor, P., \& Hillman, C.H. (2009). The persistent effects of concussion on neuroelectric indices of attention. Journal of neurotrauma, 26(9), 1463-1470.

Buzsáki, G. (2006). Rhythms of the brain. New York: Oxford University Press.

Buzzell, G.A., Barker, T.V., Troller-Renfree, S.V., Bernat, E.M., Bowers, M.E., Morales, S.,. \& Fox, N.A. (2018). Adolescent cognitive control, theta oscillations, and social motivation. bioRxiv, doi: https://doi.org/10.1101/366831

Cavanagh, J.F., Cohen, M.X., \& Allen, J.J. (2009). Prelude to and resolution of an error: EEG phase synchrony reveals cognitive control dynamics during action monitoring. The Journal of Neuroscience, 29(1), 98-105.

Cavanagh, J.F., \& Frank, M.J. (2014). Frontal theta as a mechanism for cognitive control. Trends in Cognitive Sciences, 18(8), 414421.

Cavanagh, J.F., Frank, M.J., Klein, T.J., \& Allen, J.J. (2010). Frontal theta links prediction errors to behavioral adaptation in reinforcement learning. Neuroimage, 49(4), 3198-3209.

Cavanagh, J.F., Meyer, A., \& Hajcak, G. (2017). Error-specific cognitive control alterations in generalized anxiety disorder. Biological Psychiatry. Cognitive Neuroscience and Neuroimaging, 2(5), 413-420.

Cavanagh, J.F., \& Shackman, A.J. (2015). Frontal midline theta reflects anxiety and cognitive control: Meta-analytic evidence. Journal of Physiology, Paris, 109(1), 3-15.

Cavanagh, J.F., Zambrano-Vazquez, L., \& Allen, J.J. (2012). Theta lingua franca: A common mid-frontal substrate for action monitoring processes. Psychophysiology, 49(2), 220-238.

Cohen, M.X. (2011a). Error-related medial frontal theta activity predicts cingulate-related structural connectivity. Neuroimage, 55 (3), 1373-1383.

Cohen, M.X. (2011b). It's about time. Frontiers in Human Neuroscience, $5,2$.

Cohen, M.X. (2014). Analyzing neural time series data: Theory and practice. Cambridge: MIT Press.

Cohen, M.X., \& Cavanagh, J.F. (2011). Single-trial regression elucidates the role of prefrontal theta oscillations in response conflict. Frontiers in Psychology, 2, 30.

Cohen, J., \& Cohen, P. (1983). Applied multiple regression/ correlation analysis for the behavioral sciences. Mahwah NJ: Erlbaum.

Cohen, M.X., \& van Gaal, S. (2013). Dynamic interactions between large-scale brain networks predict behavioral adaptation after perceptual errors. Cerebral Cortex, 23(5), 1061-1072.

Cook, E.W., \& Miller, G.A. (1992). Digital filtering: Background and tutorial for psychophysiologists. Psychophysiology, 29(3), $350-362$.

Coronado, V.G., McGuire, L.C., Sarmiento, K., Bell, J., Lionbarger, M.R., Jones, C.D., . . X Xu, L. (2012). Trends in traumatic brain injury in the U.S. and the public health response: 1995-2009. Journal of Safety Research, 43(4), 299-307.

De Beaumont, L., Beauchemin, M., Beaulieu, C., \& Jolicoeur, P. (2013). Long-term attenuated electrophysiological response to errors following multiple sports concussions. Journal of Clinical and Experimental Neuropsychology, 35(6), 596-607.
Debener, S., Ullsperger, M., Siegel, M., Fiehler, K., Von Cramon, D.Y., \& Engel, A.K. (2005). Trial-by-trial coupling of concurrent electroencephalogram and functional magnetic resonance imaging identifies the dynamics of performance monitoring. The Journal of Neuroscience, 25(50), 11730-11737.

Duncan, C.C., Kosmidis, M.H., \& Mirsky, A.F. (2003). Eventrelated potential assessment of information processing after closed head injury. Psychophysiology, 40(1), 45-59.

Eierud, C., Craddock, R.C., Fletcher, S., Aulakh, M., King-Casas, B., Kuehl, D., \& LaConte, S.M. (2014). Neuroimaging after mild traumatic brain injury: Review and meta-analysis. NeuroImage. Clinical, 4, 283-294.

Faul, M., Xu, L., Wald, M.M., \& Coronado, V.G. (2010). Traumatic brain injury in the United States: Emergency department visits, hospitalizations, and deaths. Atlanta: Centers for Disease Control and Prevention. National Center for Injury Prevention and Control.

Fries, P. (2005). A mechanism for cognitive dynamics: Neuronal communication through neuronal coherence. Trends in Cognitive Sciences, 9(10), 474-480.

Gehring, W.J., Goss, B., Coles, M.G., Meyer, D.E., \& Donchin, E. (1993). A neural system for error detection and compensation. Psychological Science, 4(6), 385-390.

Gehring, W.J., \& Knight, R.T. (2000). Prefrontal-cingulate interactions in action monitoring. Nature Neuroscience, 3(5), 516.

Hajcak, G., McDonald, N., \& Simons, R.F. (2003). To err is autonomic: Error-related brain potentials, ANS activity, and posterror compensatory behavior. Psychophysiology, 40(6), 895-903.

Hill, K.E., Samuel, D.B., \& Foti, D. (2016). Contextualizing individual differences in error monitoring: Links with impulsivity, negative affect, and conscientiousness. Psychophysiology, 53 (8), 1143-1153.

Hogan, A.M., Vargha-Khadem, F., Saunders, D.E., Kirkham, F.J., \& Baldeweg, T. (2006). Impact of frontal white matter lesions on performance monitoring: ERP evidence for cortical disconnection. Brain, 129(8), 2177-2188.

Iverson, G.L., Wojtowicz, M., Brooks, B.L., Maxwell, B.A., Atkins, J.E., Zafonte, R., \& Berkner, P.D. (2016). High school athletes with ADHD and learning difficulties have a greater lifetime concussion history. Journal of Attention Disorders, 1, 1-7.

Karr, J.E., Areshenkoff, C.N., \& Garcia-Barrera, M.A. (2014). The neuropsychological outcomes of concussion: A systematic review of meta-analyses on the cognitive sequelae of mild traumatic brain injury. Neuropsychology, 28(3), 321.

Kerns, J.G., Cohen, J.D., MacDonald, A.W., Cho, R.Y., Stenger, V. A., \& Carter, C.S. (2004). Anterior cingulate conflict monitoring and adjustments in control. Science, 303(5660), 1023-1026.

Kumar, S., Rao, S.L., Chandramouli, B.A., \& Pillai, S.V. (2009). Reduction of functional brain connectivity in mild traumatic brain injury during working memory. Journal of Neurotrauma, 26(5), 665-675.

Langlois, J.A., Rutland-Brown, W., \& Wald, M.M. (2006). The epidemiology and impact of traumatic brain injury: A brief overview. Journal of Head Trauma and Rehabilitation, 21(5), 375-378.

Larson, M.J., Clayson, P.E., \& Farrer, T.J. (2012). Performance monitoring and cognitive control in individuals with mild traumatic brain injury. Journal of the International Neuropsychological Society, 18(2), 323.

Larson, M.J., Farrer, T.J., \& Clayson, P.E. (2011). Cognitive control in mild traumatic brain injury: Conflict monitoring and conflict adaptation. International Journal of Psychophysiology, 82(1), 69-78.

McCrory, P., Meeuwisse, W., Johnston, K., Dvorak, J., Aubry, M., Molloy, M., \& Cantu, R. (2009). Consensus statement on 
concussion in sport 3rd International Conference on Concussion in Sport held in Zurich, November 2008. British Journal of Sports Medicine, 19(3), 185-200.

Miller, D.R., Hayes, J.P., Lafleche, G., Salat, D.H., \& Verfaellie, M. (2017). White matter abnormalities are associated with overall cognitive status in blast-related mTBI. Brain Imaging and Behavior, 11(4), 1129-1138.

Mognon, A., Jovicich, J., Bruzzone, L., \& Buiatti, M. (2011). ADJUST: An automatic EEG artifact detector based on the joint use of spatial and temporal features. Psychophysiology, 48(2), 229-240.

Moore, R.D., Pindus, D.M., Drolette, E.S., Scudder, M.R., Raine, L. B., \& Hillman, C.H. (2015). The persistent influence of pediatric concussion on attention and cognitive control during flanker performance. Biological Psychology 109, 93-102.

Narayanan, N.S., Cavanagh, J.F., Frank, M.J., \& Laubach, M. (2013). Common medial frontal mechanisms of adaptive control in humans and rodents. Nature. Neuroscience, 16(12), 1888.

Nieuwenhuis, S., Ridderinkhof, K.R., Blom, J., Band, G.P., \& Kok, A. (2001). Error-related brain potentials are differentially related to awareness of response errors: Evidence from an antisaccade task. Psychophysiology, 38(5), 752-760.

Olvet, D.M., \& Hajcak, G. (2009). Reliability of error-related brain activity. Brain Research, 1284, 89-99.

Olvet, D.M., Klein, D.N., \& Hajcak, G. (2010). Depression symptom severity and error-related brain activity. Psychiatry Research, 179(1), 30-37.

Orr, C.A., Albaugh, M.D., Watts, R., Garavan, H., Andrews, T., Nickerson, J.P., . . Hudziak, J.J. (2016). Neuroimaging biomarkers of a history of concussion observed in asymptomatic young athletes. Journal of Neurotrauma, 33(9), 803.

Pang, E.W., Dunkley, B.T., Doesburg, S.M., da Costa, L., \& Taylor, M.J. (2016). Reduced brain connectivity and mental flexibility in mild traumatic brain injury. Annals of Clinical and Translational Neurology, 3(2), 124-131.

Perrin, F., Pernier, J., Bertrand, O., Echallier, J.F., 1989. Spherical splines for scalp potential and current density mapping. Electroencephalography and Clinical Neurophysiology, 72, 184-187.

Perrin, F., Pernier, J., Bertrand, O., \& Echallier, J.F. 1990. Corrigenda. Electroencephalography and Clinical Neurophysiology, 76, 565-566.

Pontifex, M.B., O’Connor, P.M., Broglio, S.P., \& Hillman, C.H. (2009). The association between mild traumatic brain injury history and cognitive control. Neuropsychologia, 47(14), 3210-3216.

Polich, J. (2007). Updating P300: An integrative theory of P3a and P3b. Clinical Neurophysiology, 118(10), 2128-2148.

Riesel, A., Weinberg, A., Endrass, T., Meyer, A., \& Hajcak, G. (2013). The ERN is the ERN is the ERN? Convergent validity of error-related brain activity across different tasks. Biological Psychology, 93(3), 377-385.
Reches, A., Kutcher, J., Elbin, R.J., Or-Ly, H., Sadeh, B., Greer, J., . . Kontos, A.P. (2017). Preliminary investigation of Brain Network Activation (BNA) and its clinical utility in sport-related concussion. Brain Injury, 31(2), 237-246.

Rutland-Brown, W., Langlois, J.A., Thomas, K.E., \& Xi, Y.L. (2006). Incidence of traumatic brain injury in the United States, 2003. The Journal of Head Trauma Rehabilitation, 21(6), 544-548.

Smith, E.E., Reznik, S.J., Stewart, J.L., \& Allen, J.J. (2017). Assessing and conceptualizing frontal EEG asymmetry: An updated primer on recording, processing, analyzing, and interpreting frontal alpha asymmetry. International Journal of Psychophysiology, 111, 98-114.

Sponheim, S.R., McGuire, K.A., Kang, S.S., Davenport, N.D., Aviyente, S., Bernat, E.M., \& Lim, K.O. (2011). Evidence of disrupted functional connectivity in the brain after combat-related blast injury. Neuroimage, 54, S21-S29.

Stemmer, B., Segalowitz, S.J., Witzke, W., \& Schönle, P.W. (2004). Error detection in patients with lesions to the medial prefrontal cortex: An ERP study. Neuropsychologia, 42(1), 118-130.

Tsirka, V., Simos, P.G., Vakis, A., Kanatsouli, K., Vourkas, M., Erimaki, S., . . Micheloyannis, S. (2011). Mild traumatic brain injury: Graphmodel characterization of brain networks for episodic memory. International Journal of Psychophysiology, 79(2), 89-96.

Ullsperger, M., \& von Cramon, D.Y. (2006). The role of intact frontostriatal circuits in error processing. Journal of Cognitive Neuroscience, 18(4), 651-664.

Van de Vijver, I., Ridderinkhof, K.R., \& Cohen, M.X. (2011). Frontal oscillatory dynamics predict feedback learning and action adjustment. Journal of Cognitive Neuroscience, 23(12), 4106-4121.

Wessel, J.R., Klein, T.A., Ott, D.V., \& Ullsperger, M. (2014). Lesions to the prefrontal performance-monitoring network disrupt neural processing and adaptive behaviors after both errors and novelty. Cortex, 50, 45-54.

Wessel, J.R., Ullsperger, M., Obrig, H., Villringer, A., Quinque, E., Schroeter, M.L., . . . \& Klein, T.A. (2016). Neural synchrony indexes impaired motor slowing after errors and novelty following white matter damage. Neurobiology of Aging, 38, 205-213.

Wessel, J.R. (2018). An adaptive orienting theory of error processing. Psychophysiology, 55(3). doi:10.1111/psyp.13041

Zambrano-Vazquez, L., \& Allen, J.J. (2014). Differential contributions of worry, anxiety, and obsessive compulsive symptoms to ERN amplitudes in response monitoring and reinforcement learning tasks. Neuropsychologia, 61, 197-209.

Zelazo, P.D., Anderson, J.E., Richler, J., Wallner-Allen, K., Beaumont, J.L., Conway, K.P., . . . Weintraub, S. (2014). NIH Toolbox Cognition Battery (CB): Validation of executive function measures in adults. Journal of the International Neuropsychological Society, 20(6), 620-629. 